\title{
Communication \\ The Scavenging of DPPH, Galvinoxyl and ABTS Radicals by Imine Analogs of Resveratrol
}

\author{
Peter Kotora ${ }^{1, *}$, František Šeršeň ${ }^{1}$, Juraj Filo ${ }^{1}$, Dušan Loos ${ }^{1}$, Juraj Gregán̆ ${ }^{2,3, *}$ and \\ Fridrich Gregán̆ ${ }^{4}$
}

Received: 14 December 2015 ; Accepted: 14 January 2016 ; Published: 21 January 2016

Academic Editor: Derek J. McPhee

1 Institute of Chemistry, Faculty of Natural Sciences, Comenius University in Bratislava, Mlynská dolina, 84215 Bratislava, Slovakia; f.sersen@gmail.com (F.Š.); filo@fns.uniba.sk (J.F.); dusan.looos@chello.sk (D.L.)

2 Department of Genetics, Faculty of Natural Sciences, Comenius University in Bratislava, Mlynská dolina, 84215 Bratislava, Slovakia

3 Department of Chromosome Biology, MFPL, University of Vienna, Dr. Bohr-Gasse 9, 1030 Vienna, Austria

4 Department of Chemistry, Faculty of Natural Sciences, Matej Bell University, Tajovského 40, 97401 Banská Bystrica, Slovakia; Fridrich.Gregan@umb.sk

* Correspondence: kotora@fns.uniba.sk (P.K.); juraj.gregan@univie.ac.at (J.G.); Tel.: +421-2-60296-412 (P.K.); +421-2-60296-158 (J.G.)

\begin{abstract}
Resveratrol (3,5,4'-trihydroxystilbene) is a phytoalexin produced by plants. Resveratrol is known for its anti-cancer, antiviral and antioxidant properties. We prepared imine analogs of resveratrol ((hydroxyphenyliminomethyl)phenols) and tested their antioxidant activity. All prepared resveratrol analogs were able to scavenge 2,2-diphenyl-1-picrylhydrazyl (DPPH), galvinoxyl radical (GOR) and 2,2'-azino-bis(3-ethylbenzothiazoline)-6-sulphonic acid (ABTS) radicals. The antioxidant activity efficiency correlated with the number and position of hydroxyl groups. The most effective antioxidants were resveratrol analogs containing three hydroxyl groups in the benzylidene part of their molecules. These results provide new insights into the relationship between the chemical structure and biological activity of resveratrol analogs.
\end{abstract}

Keywords: resveratrol analogs; iminophenols; antioxidant activity

\section{Introduction}

The structure of (hydroxyphenyliminomethyl)phenols (Figure 1) is similar to the structure of resveratrol (3,5, $4^{\prime}$-trihydroxystilbene), which exhibits various biological activities. There are two geometric isomers of resveratrol-the trans configuration undergoes isomerisation to the cis form following exposure to ultraviolet light. Resveratrol is a phytoalexin produced by several plants that was initially identified in the flowering plant Veratrum grandiflorum. Relatively high concentrations of resveratrol are also found in grape skins and seeds, as well as red wine. Resveratrol has gained widespread attention due to its ability to extend the lifespan of various organisms, to protect against age-related diseases such as cancer and a broad range of biological activities including antiviral and antioxidant properties [1-4]. However, it is not clear whether the health benefits associated with wine drinking ("French paradox", the observation of reduced incidence of cardiovascular disease in regions of France where red wine and fats are consumed in large quantities) are due to resveratrol [5]. Molecular mechanisms underlying the health-enhancing effects of resveratrol are not well understood but some of them may be due to its antioxidant properties [6]. Although basal level of oxidative stress is important for normal cell function, severe oxidative stress often leads to oxidative damage, cell death and various diseases [7]. Resveratrol supplementation decreases the oxidative stress caused by 
consumption of a high calorie meal [8]. In addition, resveratrol is a potent activator of SIRT1, member of sirtuin family of protein deacetylases implicated in counteracting various age-related diseases [9].
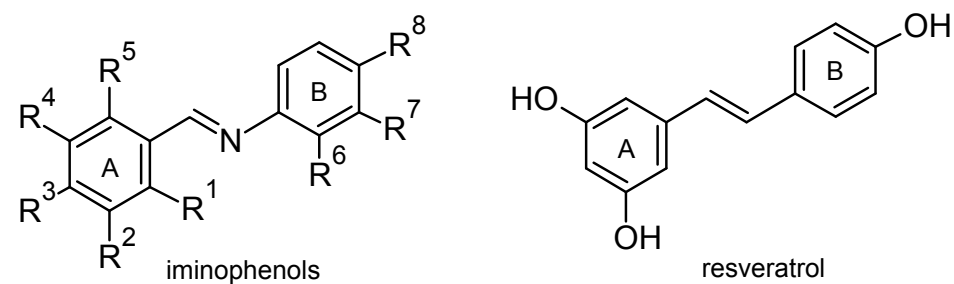

Figure 1. Structure of resveratrol and studied Hydroxyphenyliminomethyl)phenols.

Previous studies showed that modification of resveratrol could enhance its biological activities [10-12]. It is therefore important to synthesize resveratrol analogs and study their physico-chemical and biological properties. Imines are often used as ligands for the complexation of metals; they are used for the preparation of liquid crystals and are also used in analytical and pharmaceutical chemistry [13]. The traditional method of the imine preparation is based on heating aldehydes with amines in an organic solvent (benzene or toluene) [13-15]. Synthesis in which the organic solvent is replaced with water [16] or other recycle medium such as polypropylene glycol [17] has been described as "green" alternative to these preparations.

The goal of this work was to prepare various imine analogs of resveratrol (some of which have not been described in the literature yet) and to test their antioxidant activity. We analyzed antioxidant properties of twenty-one (hydroxyphenyliminomethyl)phenols (Figure 1) where $\mathrm{R}^{\mathrm{i}}=\mathrm{OH}$ or $\mathrm{H}$.

The studied (hydroxyphenyliminomethyl)phenols consist of two parts; one is aminophenol (AP) and the other is phenolic part from hydroxybenzaldehyde (HBA). Some of these compounds are produced as secondary metabolites in plants. 4-HBA and 3,4-dihydroxy-benzaldehyde (DHBA) are present in Origanum vulgare L. [18], oil palm biomass [19], Potentilla fulgens [20], sugarcane molasses [21] and vanilla pods [22]. 4-HBA and 4-AP are present in Dilobeia thouarsii [23]. Many HBAs are known for their flavoring and antimicrobial properties [24,25]. Antioxidant properties of some HBAs (2-HBA, 3-HBA, 3,4-DHBA) were studied by the Tsimidou group [26,27]. Some of imine resveratrol anologs were studied as multifunctional drugs in the treatment of Alzheimer's dissease [28] and they also exhibit antioxidant activity [28,29]. Some hydroxybenzene diimine derivatives possess important biological activities such as antioxidant [30], antiinflammatory and anti-analgesics [31].

\section{Results and Discussion}

\subsection{Chemistry}

The starting compounds for preparation of (hydroxyphenyliminomethyl)phenols were mono aminophenols (APs) and mono-, di- and trihydroxybenzaldehydes (HBAs). Twenty-one (hydroxy phenyliminomethyl)phenols (compounds 1-21, for full names see Section 3.2. Synthesis) were prepared by condensation reactions where the first step was addition of amino group on carbonyl group followed by elimination of water according to protocols of Tanaka and Shiraishi [16] and Lu et al. [29] (Scheme 1). Briefly, equimolar amounts of appropriate AP and HBA were stirred in a small amount of distilled water at room temperature $\left(25^{\circ} \mathrm{C}\right)$ for $2 \mathrm{~h}$. Obtained products were filtered, washed several times with distilled water and dried at $45^{\circ} \mathrm{C}$ in vacuum. Yields of (hydroxyphenyliminomethyl)phenols ranged between $26 \%$ and $95 \%$. The compounds were characterized by melting point, elemental analysis, ${ }^{1} \mathrm{H}$ - and ${ }^{13} \mathrm{C}-\mathrm{NMR}, \mathrm{IR}$ and MS spectra. 
<smiles>[R]c1c([R])c([R])c(C=O)c([R])c1[R]</smiles>

Scheme 1. Preparation of iminophenol analogs of resveratrol.

\subsection{Antioxidant Activity}

All prepared (hydroxyphenyliminomethyl)phenols 1-21 were able to scavenge 2,2-diphenyl-1picrylhydrazyl (DPPH), galvinoxyl and 2,2'-azino-bis(3-ethylbenzothiazoline)-6-sulphonic acid (ABTS) radicals (Table 1). This table shows that some of the prepared (hydroxyphenyliminomethyl)phenols were more effective scavengers of DPPH (compounds: $8,10,12,14,17-21$ ) or galvinoxyl (GOR) (compounds 2, 9, 13, 16, 17-21) as compared to resveratrol (Table 1). On the other hand all prepared (hydroxyphenyliminomethyl)phenols exhibited lower efficiency of ABTS scavenging as resveratrol, their $\mathrm{SC}_{50}$ values ranged from 1.98 to $18.16 \mu \mathrm{mol} / \mathrm{dm}^{3}$.

Table 1. $\mathrm{SC}_{50}$ and proton affinity (PA) + electron transfer enthalpy (ETE) values of studied (hydroxy phenyliminomethyl)phenols.

\begin{tabular}{|c|c|c|c|c|c|}
\hline Compound & $\begin{array}{c}\text { DPPH SC }{ }_{50} / \mathrm{r}^{2} \\
\left(\mu \mathrm{mol} / \mathrm{dm}^{3}\right)\end{array}$ & $\begin{array}{c}\text { GOR SC }_{50} / \mathrm{r}^{2} \\
\left(\mu \mathrm{mol} / \mathrm{dm}^{3}\right)\end{array}$ & $\begin{array}{c}\text { ABTS } \mathrm{SC}_{50} / \mathrm{r}^{2} \\
\left(\mu \mathrm{mol} / \mathrm{dm}^{3}\right)\end{array}$ & $\begin{array}{c}\text { PA + ETE in } \\
\text { Methanol (kJ/mol) }\end{array}$ & $\begin{array}{c}\text { PA + ETE in } \\
\text { Water }(\mathrm{kJ} / \mathrm{mol})\end{array}$ \\
\hline 1 & $27.90 / 0.951$ & $184 / 0.985$ & $11.64 / 0.969$ & 546.8 & 562.2 \\
\hline 2 & $38.26 / 0.963$ & $48.27 / 0.998$ & $8.50 / 0.809$ & 550.9 & 566.0 \\
\hline 3 & $560 / 0.893$ & $3075 / 0.809$ & $8.50 / 0.928$ & 547.7 & 562.2 \\
\hline 4 & $967 / 0.976$ & $415 / 0.995$ & $3.54 / 0.993$ & 565.4 & 580.7 \\
\hline 5 & $383 / 0.936$ & $393 / 0.903$ & $6.74 / 0.869$ & 547.1 & 562.1 \\
\hline 6 & $88.64 / 0.974$ & $251 / 0.999$ & $3.86 / 0.994$ & 550.1 & 564.4 \\
\hline 7 & $53.98 / 0.937$ & $184 / 0.979$ & $6.57 / 954$ & 547.4 & 562.8 \\
\hline 8 & $21.00 / 0.997$ & $127 / 0.971$ & $14.39 / 0.972$ & 543.1 & 551.9 \\
\hline 9 & $43.00 / 0.991$ & $39.96 / 0.945$ & $18.16 / 0.922$ & 520.8 & 537.4 \\
\hline 10 & $19.00 / 0.994$ & $2300 / 0.987$ & $6.4 / 0.988$ & 539.6 & 554.7 \\
\hline 11 & $83.00 / 0.947$ & $73.78 / 0.998$ & $3.05 / 0.847$ & 554.6 & 569.9 \\
\hline 12 & $24.00 / 0.970$ & $456 / 0.995$ & $3.31 / 0.968$ & 541.8 & 554.0 \\
\hline 13 & $18.00 / 0.988$ & $23.34 / 0.997$ & $6.40 / 0.977$ & 542.7 & 557.6 \\
\hline 14 & $12.52 / 0.964$ & $102 / 0.865$ & $5.83 / 0.988$ & 547.5 & 573.9 \\
\hline 15 & $42.00 / 0.986$ & $55.43 / 0.727$ & $3.74 / 0.918$ & 540.3 & 555.4 \\
\hline 16 & $149 / 0.979$ & 173/0.988 & $2.53 / 0.962$ & 554.9 & 570.2 \\
\hline 17 & $22.05 / 0.988$ & $25.24 / 0.989$ & $2.01 / 0.989$ & 537.4 & 552.7 \\
\hline 18 & $12.60 / 0.996$ & $27.67 / 0.993$ & $2.83 / 0.956$ & 525.8 & 540.7 \\
\hline 19 & $9.05 / 0.988$ & $72.10 / 0.958$ & $2.46 / 0.977$ & 531.1 & 546.5 \\
\hline 20 & $18.05 / 0.988$ & $23.92 / 0.985$ & $2.92 / 0.9997$ & 548.4 & 563.1 \\
\hline 21 & $8.77 / 0.943$ & $15.39 / 0.994$ & $1.98 / 0.994$ & 528.5 & 544.0 \\
\hline Resveratrol & $26.37 / 0.849$ & $72.66 / 0.910$ & $1.43 / 0.959$ & 548.6 & 563.5 \\
\hline
\end{tabular}

$\mathrm{r}^{2}$ is an average square deviation.

Antioxidant activities of compounds 1, 2, 3, 4, 5, 7, 9 and 16 have already been studied [28,29]. $\mathrm{SC}_{50}$ values reported by Lu et al. [29] and $\mathrm{Li}$ et al. [28] are similar to those presented in our current study. However, our melting points NMR spectra of compounds $\mathbf{2}$ and $\mathbf{9}$ do not correspond with those reported by Li et al. [28]. The chemical shift for the compound 2 published by $\mathrm{Li}$ et al. [30] for $\mathrm{OH}$ (in meta position of ring A) $\delta=14.21$ is too high and $\delta=9.63$ for compound 9 is too small. This raises the possibility that compounds 2 and 9 prepared by Li et al. [28] are not identical to the denoted structures.

We found that the efficiency of the radical scavenging correlated with the position and number of hydroxyl groups in prepared compounds. Regarding the position of the hydroxyl group in hydroxyphenylimino parts of prepared compounds, most (hydroxyphenyliminomethyl)phenols with the $\mathrm{OH}$ group in ortho position of the ring A were the most potent scavengers of DPPH and galvinoxyl radicals. (Hydroxyphenyliminomethyl)phenols with three $\mathrm{OH}$ groups in the ring A were 
the most potent scavengers of ABTS radicals. (Hydroxyphenyliminomethyl)phenols 2, 4, 6, 7, 11, 16 which contained one or two $\mathrm{OH}$ groups in positions 3 and 4 of the ring A exhibited relatively low ability to scavenge DPPH or GOR radicals (their values of $\mathrm{SC}_{50}$ ranged from 38 to $3075 \mu \mathrm{mol} / \mathrm{dm}^{3}$ ). However, compounds 3 and 5, which have $\mathrm{OH}$ group in the ortho position of the benzene ring $\mathrm{A}$, exhibited low antioxidant activity. On the other hand, compunds 17 and 21, which do not have $\mathrm{OH}$ groups in the ortho position of the phenyl ring A, exhibited high antioxidant activity.

Based on the ${ }^{1} \mathrm{H}-\mathrm{NMR}$ spectra analyses, we assumed that proton could be most easily dissociation from the $\mathrm{OH}$ group in the ortho position of the ring A. (Hydroxyphenyliminomethyl)phenols with $\mathrm{R}^{1}=\mathrm{OH}$ could form intramolecular hydrogen bond with the nitrogen of imine group. Due to this interaction acidity of hydrogen at this position increased, which was confirmed by the high chemical shift ( $\delta=12.25$ to $14.19 \mathrm{ppm}$ ). On the other hand, the chemical shift of the $\mathrm{OH}$ protons bound in the meta or para position were $<8.10 \mathrm{ppm}$.

It is generally assumed that the mechanism of radical scavenging by phenolic compounds is associated with the ability to release hydrogen atom, proton or electron from the antioxidant molecule. We speculated that releasing of the proton from $\mathrm{OH}$ group could be the main mechanism respossible for the antioxidant activity of studied (Hydroxyphenyliminomethyl)phenols. Consistent with this assumption were the $\mathrm{SC}_{50}$ values of studied compounds with higher $\delta$ which exhibited higher antioxidant activity as compared to other (Hydroxyphenyliminomethyl)phenols.

To find a possible correlation between the antioxidant activity of (hydroxyphenyliminome thyl)phenols and a position of the hydroxyl groups, we used the method PM6 (see the Experimental Section) to calculate the energy associated with the release of hydrogen (bond dissociation enthalpy, $\mathrm{BDE}$ ); the proton relaxation (PDE); release of electron (IP ionization potential) and the energy associated with combined transfer of the electron and proton (ETE and PA, respectively) for the studied (Hydroxyphenyliminomethyl)phenols. The values of these energies in methanol or in water are presented in Tables S1-S8.

The antioxidant mechanism associated with the release of a hydrogen atom can be described by the Equation (1). From the point of view of thermodynamics the strength of the antioxidant effect in the model describes BDE (bond dissociation enthalpy, Equation (2)):

$$
\begin{gathered}
\mathrm{ArOH}+\mathrm{R}^{\bullet} \rightarrow \mathrm{ArO}^{\bullet}+\mathrm{R}^{\bullet} \\
\mathrm{BDE}=\mathrm{H}\left(\mathrm{ArO}^{\bullet}\right)+\mathrm{H}^{\bullet}\left(\mathrm{H}^{\bullet}\right)-\mathrm{H}(\mathrm{Ar}-\mathrm{OH})
\end{gathered}
$$

The other antioxidant mechanism of (hydroxyphenyliminomethyl)phenols may be associated with combined transfer of electrons and protons to radical $\left(R^{\bullet}\right)$. In the first step, the electron is transferred to the radical (Equation (3)). The possibility of such transition is determined by the size of ionization potential of IP, which describes the Equation (4):

$$
\begin{gathered}
\mathrm{ArOH}+\mathrm{R}^{\bullet} \rightarrow \mathrm{Ar}-\mathrm{OH}^{\bullet+}+\mathrm{R}^{-} \\
\mathrm{IP}=\mathrm{H}\left(\mathrm{Ar}-\mathrm{OH}^{\bullet+}\right)+\mathrm{H}\left(\mathrm{e}^{-}\right)-\mathrm{H}(\mathrm{Ar}-\mathrm{OH})
\end{gathered}
$$

In the second step, a proton is transferred to the anion radical $\mathrm{R}^{-}$(Equation (5)). The possibility of such a transfer is characterized by the value of PDE (proton dissotiation enthalpy, Equation (6)):

$$
\begin{gathered}
\mathrm{Ar}-\mathrm{OH}^{\bullet+}+\mathrm{R}^{-} \rightarrow \mathrm{ArO}^{\bullet}+\mathrm{RH} \\
\mathrm{PDE}=\mathrm{H}\left(\mathrm{ArO}^{\bullet}\right)+\mathrm{H}\left(\mathrm{H}^{+}\right)-\mathrm{H}\left(\mathrm{Ar}-\mathrm{OH}^{\bullet+}\right)
\end{gathered}
$$

Another possible mechanism of the antioxidant effect of (hydroxyphenyliminomethyl)phenols is the gradual loss of a proton followed by electron transfer (Equations (7) and (8)):

$$
\mathrm{ArOH} \rightarrow \mathrm{ArO}^{-}+\mathrm{H}^{+}
$$




$$
\begin{gathered}
\mathrm{ArO}^{-}+\mathrm{R}^{\bullet} \rightarrow \mathrm{ArO}^{\bullet}+\mathrm{R}^{-} \\
\mathrm{R}^{-}+\mathrm{H}^{+} \rightarrow \mathrm{RH}
\end{gathered}
$$

The reaction enthalpy of the first step (Equation (7)) corresponds to the proton affinity PA (Equation (10)) whereas the reaction enthalpy of the second step (Equation (8)) describes enthalpy of electron transfer (ETE, Equation (11)):

$$
\begin{gathered}
\mathrm{PA}=\mathrm{H}\left(\mathrm{ArO}^{-}\right)+\mathrm{H}\left(\mathrm{H}^{+}\right)-\mathrm{H}(\mathrm{Ar}-\mathrm{OH}) \\
\mathrm{ETE}=\mathrm{H}\left(\mathrm{ArO}^{\bullet}\right)+\mathrm{H}\left(\mathrm{e}^{-}\right)-\mathrm{H}\left(\mathrm{ArO}^{-}\right)
\end{gathered}
$$

Comparison of enthalpies required for the above mentioned antioxidant reactions suggests that the preferred mechanism seems to be the one described by Equations (7)-(11), those characterized by the values of PA + ETE. Based on the above mentioned ideas, we speculated that the lower PA + ETE values of studied (hydroxyphenyliminomethyl)phenols may imply their higher antioxidant activity, i.e., lower value of $\mathrm{SC}_{50}$. Figure 2 shows the dependence of $\mathrm{SC}_{50}$ scavenging DPPH radicals on the sum of PA + ETE enthalpy. As can be seen from these figures, there is no obvious relationship between these parameters. This discrepancy could be caused by forming of (hydroxyphenyliminomethyl)phenols self-aggregates which could reduce the antioxidant activity of original compounds.
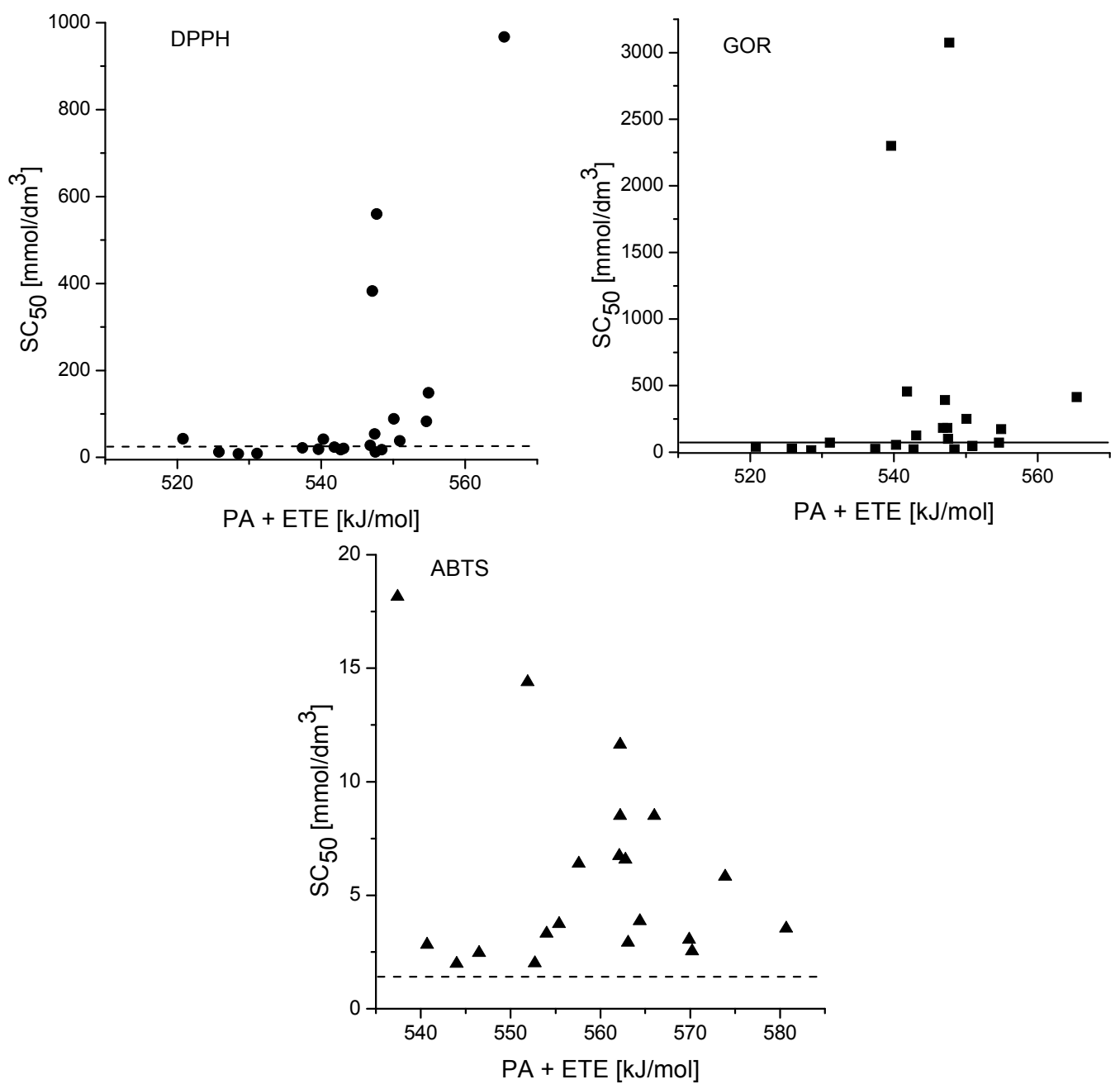

Figure 2. Dependence of $\mathrm{SC}_{50}$ of DPPH, GOR and ABTS scavenging by studied (Hydroxypheny liminomethyl)phenols on PA + ETE entalpies. Dashed lines denote the $\mathrm{SC}_{50}$ values of resveratrol. 
To test this assumption we performed the following experiment. According to the work of Cigáň et al. [32], creation of self-aggregates can be determined from concentration-dependence of absorption or fluorescence spectra. The formation of self-aggregates should be manifested by a shift of emission maxima or by a shape change in absorption/fluorescence spectra with an increase of concentration of the studied compound. We observed that the corresponding changes in absorption spectra were very small. Also in fluorescence emission spectra, relatively small batochromic shifts were observed with increasing concentrations. Compound 5 with lower antioxidant activity exhibited approximately $11 \mathrm{~nm}$, whereas compound 14 with higher antioxidant activity showed only $4 \mathrm{~nm}$ (data not shown). The formation of self-aggregates was apparent from the fluorescence excitation spectra. We observed changes in shapes of these spectra with the increasing concentrations of (Hydroxyphenyliminomethyl)phenols (Figure 3). These results suggest that one possible explanation for the lower antioxidant activity of some of the studied (Hydroxyphenyliminomethyl)phenols is formation of self-aggregates. A possible structure of aggregates for compound 3 is presented in Figure S1.
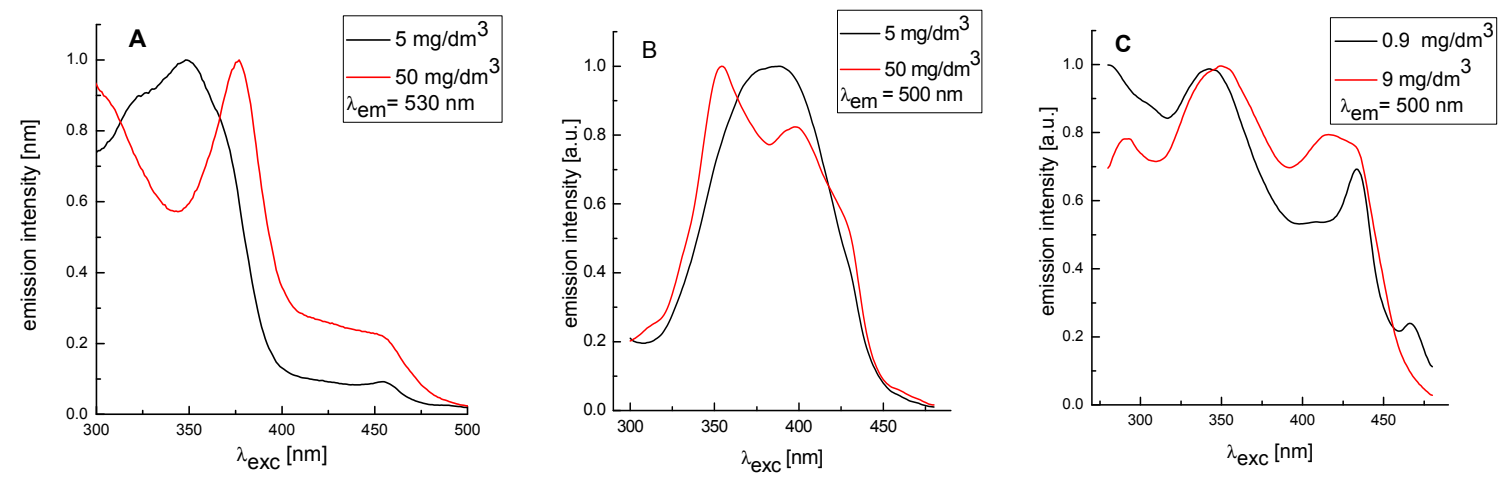

Figure 3. Fluorescence excitation spectra of studied (Hydroxyphenyliminomethyl)phenols: 5 (A); 14 (B) and $15(C)$ in methanol at concentration of $0.9 \mathrm{mg} / \mathrm{dm}^{3}$. The excitation and emission slits were 2 resp. $10 \mathrm{~nm}$.

Numerous studies showed that various modifications of resveratrol molecule enhance its biological activities including the antioxidant properties [10-12,33]. It is therefore important to synthesize new resveratrol analogs and study their physico-chemical and biological properties. We aimed to identify novel analogues of resveratrol that could potently scavenge radicals and serve as leads for the development of future drugs. To find out more about potential pharmacological and nutritional relevance of (Hydroxyphenyliminomethyl)phenols synthesized in our current study, it will be important to test in vivo activities of these compounds. However, given their unstability in water, additional chemical modifications may be required to achieve high in vivo activity. Our current study brings new insights into the relationship between the chemical structure and antioxidant properties of resveratrol analogs.

\section{Experimental Section}

\subsection{General Information}

Methyl alcohol p.a. was purchased from Centralchem (Bratislava, Slovakia). 2-Aminophenol, 3-amino-phenol, 4-aminophenol, 2-hydroxybenzaldehyde, 3-hydroxybenzaldehyde, 4-hydroxybenzaldehyde, 2,3-dihydroxybenzaldehyde, 2,4-dihydroxybenzaldehyde, 2,5-dihydroxybenzaldehyde, 3,4-dihydroxybenzaldehyde, 3,5-dihydroxybenzaldehyde, 2,3,4-trihydroxybenzaldehyde, 2,4,6-trihyd roxybenzaldehyde, 3,4,5-trihydroxybenzaldehyde, 2,2-diphenyl-1-picrylhydrazyl, were purchased from Alfa Aesar (Heysham, UK). 2,2'-Azino-bis(3-ethylbenzothiazoline-6-sulfonic acid) diammonium salt (ABTS) was purchased from Sigma-Aldrich (St. Louis, MO, USA). 
Absorption spectra were recorded by a Genesis 6 spectrophotometer (Thermo-Scientific, Waltham, MA, USA). Fluorescence emission spectra were recorded by a FSP 920 spectrofluorimeter (Edinburgh Instruments, Livingston, UK) and ${ }^{1} \mathrm{H}-(300 \mathrm{MHz})$ and ${ }^{13} \mathrm{C}-\mathrm{NMR}(75 \mathrm{MHz})$ spectra were registered on a VNMRS 300 spectrometer (Varian, Salt Lake City, UT, USA) in DMSO- $\mathrm{d}_{6}$ with tetramethylsilane as internal standard. Melting points were determined on a Kofler hot plate apparatus and are uncorrected. Elemental analyses were obtained on an Elemental Analyzer CHNS-OEA 1108 (Carlo Erba, Wigan, UK). FTIR spectra (in solid phase) were recorded on a Nicolet 6700 spectrometer (Thermo-Scientific (Nicolet), Waltham, MA, USA) using the ATR technique.

MS spectra were recorded by a LC-MS spectrometer consisting of an Agilent 1200 HPLC (Walbron, Germany), with a MSD 6110 MS detector (Agilent Technologies, Santa Clara, CA, USA).

\subsection{Synthesis}

General Procedure for the Synthesis of (Hydroxyphenyliminomethyl)phenols

Twenty-one (hydroxyphenyliminomethyl)phenols were prepared following the protocol described by Tanaka and Shiraishi [16]. Briefly, equimolar amounts of mono-, di- and tri- hydroxy benzaldehydes (HBA) and 2-, 3- or 4-aminophenols (AP) were stirred (2 h) in distilled water at room temperature $\left(25^{\circ} \mathrm{C}\right)$. Obtained products were filtered, washed by water and dried at $45^{\circ} \mathrm{C}$. Yields of (Hydroxyphenyliminomethyl)phenols ranged between 36\%-95\%. The prepared compounds were characterized by melting point, elemental analysis, ${ }^{1} \mathrm{H}$ - and ${ }^{13} \mathrm{C}-\mathrm{NMR}$, IR and MS spectra.

3-\{[(2-Hydroxyphenyl)imino]methyl\}phenol (1): Yield 77\%; m.p. 182-184 ${ }^{\circ}$ C. Anal. Calc. for $\mathrm{C}_{13} \mathrm{H}_{11} \mathrm{NO}_{2}$ (213.24) C, 73.22; H, 5.20; N, 6.57. Found: C, 73.43; H, 5.04; N, 6.74\%. IR: $1627.4(v / C=N) .{ }^{1} \mathrm{H}-\mathrm{NMR}$ $\left(\mathrm{DMSO}-d_{6}\right) \delta: 13.77(\mathrm{~s}, 1 \mathrm{H}), 9.71(\mathrm{~s}, 1 \mathrm{H}), 8.96(\mathrm{~s}, 1 \mathrm{H}), 7.61(\mathrm{dd}, J=7.9,1.3 \mathrm{~Hz}, 1 \mathrm{H}), 7.46-7.27(\mathrm{~m}, 2 \mathrm{H})$, 7.12 (ddd, 1H), 7.03-6.80 (m, 4H). ${ }^{13} \mathrm{C}-\mathrm{NMR}$ (DMSO-d 6 ) $\delta:$ 162.10, 161.16, 151.56, 135.36, 133.26, 132.74, $128.49,120.03,120.00,119.93,119.16,117.12,116.95$; positive LC-MS m/z: $214.1[\mathrm{M}+\mathrm{H}]^{+}$calc. for $\mathrm{C}_{13} \mathrm{H}_{12} \mathrm{NO}_{2}{ }^{+}, 214.09$, found 214.1

2-\{[(3-Hydroxyphenyl)imino]methyl\}phenol (2): Yield 71\%; m.p. 116-118 ${ }^{\circ} \mathrm{C}$. Anal. Calc. for $\mathrm{C}_{13} \mathrm{H}_{11} \mathrm{NO}_{2}$ (213.24) C, 73.22; H, 5.20; N, 6.57. Found: C, 73.47; H, 5.09; N, 6.50\%. IR: $1619.9(v / C=N) .{ }^{1} \mathrm{H}-\mathrm{NMR}$ $\left(\mathrm{DMSO}_{6}\right) \delta: 9.64(\mathrm{~s}, 1 \mathrm{H}), 8.97(\mathrm{~s}, 1 \mathrm{H}), 8.58(\mathrm{~s}, 1 \mathrm{H}), 7.41(\mathrm{~d}, J=7.8 \mathrm{~Hz}, 2 \mathrm{H}), 7.30(\mathrm{dd}, J=7.7 \mathrm{~Hz}, 1 \mathrm{H}), 7.14$ $(\mathrm{d}, J=7.7 \mathrm{~Hz}, 1 \mathrm{H}), 7.07(\mathrm{dd}, J=7.0 \mathrm{~Hz}, 1 \mathrm{H}), 7.00-6.73(\mathrm{~m}, 3 \mathrm{H}) .{ }^{13} \mathrm{C}-\mathrm{NMR}\left(\mathrm{DMSO}-d_{6}\right) \delta: 160.04,158.02$, $151.35,138.64,138.16,130.09,127.58,120.77,119.89,119.81,118.91,116.40,115.05$; positive LC-MS $m / z$ : $214.1[\mathrm{M}+\mathrm{H}]^{+}$calc. for $\mathrm{C}_{13} \mathrm{H}_{12} \mathrm{NO}_{2}{ }^{+}, 214.09$, found 214.1 .

2-\{[(3-Hydroxyphenyl)imino]methyl\}phenol (3): Yield 69\%; m.p. 119-120 ${ }^{\circ}$ C. Anal. Calc. for $\mathrm{C}_{13} \mathrm{H}_{11} \mathrm{NO}_{2}$ (213.24) C, 73.22; H, 5.20; N, 6.57. Found: C, 73.10; H, 5.13; N, 6.51\%. IR: $1602.8(v / C=N) .{ }^{1} \mathrm{H}-\mathrm{NMR}$ $\left(\mathrm{DMSO}-d_{6}\right) \delta: 13.12(\mathrm{~s}, 1 \mathrm{H}), 9.64(\mathrm{~s}, 1 \mathrm{H}), 8.90(\mathrm{~s}, 1 \mathrm{H}), 7.65(\mathrm{dd}, J=7.6,1.5 \mathrm{~Hz}, 1 \mathrm{H}), 7.41(\mathrm{ddd}, J=8.2$, $1.6 \mathrm{~Hz}, 1 \mathrm{H}), 7.25(\mathrm{dd}, J=7.9 \mathrm{~Hz}, 1 \mathrm{H}), 6.97(\mathrm{dd}, J=8.3 \mathrm{~Hz}, 2 \mathrm{H}), 6.83(\mathrm{~d}, J=7.8 \mathrm{~Hz}, 1 \mathrm{H}), 6.77(\mathrm{dd}, J=2.0 \mathrm{~Hz}$, $1 \mathrm{H}), 6.73(\mathrm{dd}, J=8.0,1.7 \mathrm{~Hz}, 1 \mathrm{H}) .{ }^{13} \mathrm{C}-\mathrm{NMR}\left(\mathrm{DMSO}-d_{6}\right) \delta: 163.64,160.74,158.75,149.70,133.65,133.02$, $130.62,119.66,119.52,116.99,114.51,112.49,108.56$; positive LC-MS m/z: $214.1[\mathrm{M}+\mathrm{H}]^{+}$calc. for $\mathrm{C}_{13} \mathrm{H}_{12} \mathrm{NO}_{2}{ }^{+}, 214.09$, found 214.1 .

3-\{[(4-Hydroxyphenyl)imino]methyl\}phenol (4): Yield 78\%; m.p. 210-212 ${ }^{\circ}$ C. Anal. Calc. for $\mathrm{C}_{13} \mathrm{H}_{11} \mathrm{NO}_{2}$ (213.24) C, 73.22; H, 5.20; N, 6.57. Found: C, 73.38; H, 5.23; N, 6.62\%. IR: $1625.5(v / C=N) .{ }^{1} \mathrm{H}-\mathrm{NMR}$ $\left(\mathrm{DMSO}_{-} \mathrm{d}_{6}\right) \delta: 10.09(\mathrm{~s}, 1 \mathrm{H}), 9.44(\mathrm{~s}, 1 \mathrm{H}), 8.39(\mathrm{~s}, 1 \mathrm{H}), 7.75(\mathrm{~d}, J=8.4 \mathrm{~Hz}, 2 \mathrm{H}), 7.15(\mathrm{t}, J=7.9 \mathrm{~Hz}, 1 \mathrm{H}), 6.87$ $(\mathrm{d}, J=8.4 \mathrm{~Hz}, 2 \mathrm{H}), 6.60$ (dd, 3H). ${ }^{13} \mathrm{C}-\mathrm{NMR}\left(\mathrm{DMSO}-\mathrm{d}_{6}\right) \delta: 161.00,160.01,158.49,153.82,131.07,130.22$, 127.94, 116.07, 112.86, 112.02, 108.19; positive LC-MS m/z: $214.1[\mathrm{M}+\mathrm{H}]^{+}$calc. for $\mathrm{C}_{13} \mathrm{H}_{12} \mathrm{NO}_{2}{ }^{+}, 214.09$, found 214.1 .

2-\{[(4-Hydroxyphenyl)imino]methyl\}phenol (5): Yield 38\%; m.p. 138-139 ${ }^{\circ}$ C. Anal. Calc. for $\mathrm{C}_{13} \mathrm{H}_{11} \mathrm{NO}_{2}$ (213.24) C, 73.22; H, 5.20; N, 6.57. Found: C, 73.40; H, 4.98; N, 6.49\%. IR: $1614.3(v / C=N) .{ }^{1} \mathrm{H}-\mathrm{NMR}$ $\left(\mathrm{DMSO}-d_{6}\right) \delta: 13.42(\mathrm{~s}, 1 \mathrm{H}), 9.67(\mathrm{~s}, 1 \mathrm{H}), 8.90(\mathrm{~s}, 1 \mathrm{H}), 7.59$ (dd, J = 7.6, $\left.1.4 \mathrm{~Hz}, 1 \mathrm{H}\right), 7.47-7.21(\mathrm{~m}, 3 \mathrm{H})$, 
7.08-6.89 (m, 2H), $6.86(\mathrm{dd}, J=5.9 \mathrm{~Hz}, 2 \mathrm{H}) .{ }^{13} \mathrm{C}-\mathrm{NMR}\left(\mathrm{DMSO}-d_{6}\right) \delta: 160.61,160.58,157.40,139.61$, $132.93,132.63,123.08,119.86,119.39,116.89,116.39$; positive LC-MS $m / z: 214.1[\mathrm{M}+\mathrm{H}]^{+}$calc. for $\mathrm{C}_{13} \mathrm{H}_{12} \mathrm{NO}_{2}{ }^{+}, 214.09$, found 214.1.

3-\{[(4-Hydroxyphenyl)imino]methyl\}phenol (6): Yield 84\%; m.p. $172-173{ }^{\circ} \mathrm{C}$. Anal. Calc. for $\mathrm{C}_{13} \mathrm{H}_{11} \mathrm{NO}_{2}$ (213.24) C, 73.22; H, 5.20; N, 6.57. Found: C, 73.04; H, 5.25; N, 6.41\%. IR: 1622.4. (v/C=N), ${ }_{1}^{1} \mathrm{H}-\mathrm{NMR}$ (DMSO- $\left.d_{6}\right) \delta: 9.62(\mathrm{~s}, 1 \mathrm{H}), 9.48(\mathrm{~s}, 1 \mathrm{H}), 8.51(\mathrm{~s}, 1 \mathrm{H}), 7.41-7.23(\mathrm{~m}, 3 \mathrm{H}), 7.18(\mathrm{~d}, J=8.7 \mathrm{~Hz}, 2 \mathrm{H}), 6.99-6.84$ $(\mathrm{m}, 1 \mathrm{H}), 6.80(\mathrm{~d}, J=8.7 \mathrm{~Hz}, 2 \mathrm{H}) .{ }^{13} \mathrm{C}-\mathrm{NMR}\left(\mathrm{DMSO}_{6}\right)$ ) $8: 157.67,157.18,156.27,142.59,137.86,129.78$, 122.51, 119.99, 118.19, 115.74, 113.89; positive LC-MS $m / z: 214.1[\mathrm{M}+\mathrm{H}]^{+}$calc. for $\mathrm{C}_{13} \mathrm{H}_{12} \mathrm{NO}_{2}{ }^{+}, 214.09$, found 214.1.

4-\{[(4-Hydroxyphenyl)imino]methyl\}phenol (7): Yield 82\%; m.p. $183-185^{\circ} \mathrm{C}$. Anal. Calc. for $\mathrm{C}_{13} \mathrm{H}_{11} \mathrm{NO}_{2}$ (213.24) C, 73.22; H, 5.20; N, 6.57. Found: C, 73.48; H, 5.02; N, 6.33\%. IR: 1639.6 (v/C=N). ${ }^{1} \mathrm{H}-\mathrm{NMR}$ (DMSO- $\left.d_{6}\right) \delta: 10.00(\mathrm{~s}, 1 \mathrm{H}), 9.37(\mathrm{~s}, 1 \mathrm{H}), 8.44(\mathrm{~s}, 1 \mathrm{H}), 7.72(\mathrm{~d}, J=8.6 \mathrm{~Hz}, 2 \mathrm{H}), 7.11(\mathrm{~d}, J=8.7 \mathrm{~Hz}, 2 \mathrm{H})$, $6.86(\mathrm{~d}, J=8.6 \mathrm{~Hz}, 2 \mathrm{H}), 6.77(\mathrm{~d}, J=8.7 \mathrm{~Hz}, 2 \mathrm{H}) .{ }^{13} \mathrm{C}-\mathrm{NMR}\left(\mathrm{DMSO}-d_{6}\right) \delta: 160.10,156.89,155.68,143.21$, 130.18, 127.91, 122.15, 115.64, 115.58; positive LC-MS $m / z$ : $214.1[\mathrm{M}+\mathrm{H}]^{+}$calc. for $\mathrm{C}_{13} \mathrm{H}_{12} \mathrm{NO}_{2}{ }^{+}, 214.09$, found 214.1.

3-\{[(2-Hydroxyphenyl)imino]methyl\}benzene-1,2-diol (8): Yield 75\%; m.p. 168-169 ${ }^{\circ} \mathrm{C}$. Anal. Calc. for $\mathrm{C}_{13} \mathrm{H}_{11} \mathrm{NO}_{3}$ (229.24) C, 68.11; H, 4.84; N, 6.11. Found: C, 68.29; H, 5.06; N, 6.14 \%. IR: $1627.7(v / \mathrm{C}=\mathrm{N})$. ${ }^{1} \mathrm{H}-\mathrm{NMR}$ (DMSO-d $\left.d_{6}\right) \delta: 14.19(\mathrm{~s}, 1 \mathrm{H}), 9.83(\mathrm{~s}, 1 \mathrm{H}), 9.02(\mathrm{~s}, 1 \mathrm{H}), 8.94(\mathrm{~s}, 1 \mathrm{H}), 7.41(\mathrm{dd}, J=7.8,1.5 \mathrm{~Hz}, 1 \mathrm{H})$, $7.13(\mathrm{ddd}, J=7.8,1.5 \mathrm{~Hz}, 1 \mathrm{H}), 7.04(\mathrm{dd}, J=7.8,1.5 \mathrm{~Hz}, 1 \mathrm{H}), 6.98(\mathrm{dd}, J=8.1,1.2 \mathrm{~Hz}, 1 \mathrm{H}), 6.94-6.85$ $(\mathrm{m}, 2 \mathrm{H}), 6.72(\mathrm{dd}, J=7.8 \mathrm{~Hz}, 1 \mathrm{H}) .{ }^{13} \mathrm{C}-\mathrm{NMR}\left(\mathrm{DMSO}-d_{6}\right) \delta: 161.63,152.02,151.29,146.49,134.38,128.40$, $122.89,120.06,119.60,119.41,118.47,118.30,116.91$; positive LC-MS $m / z: 230.1[\mathrm{M}+\mathrm{H}]^{+}$calc. for $\mathrm{C}_{13} \mathrm{H}_{12} \mathrm{NO}_{3}{ }^{+}, 230.08$, found 230.1 .

4-\{[(2-Hydroxyphenyl)imino]methyl\}benzene-1,3-diol (9): Yield 77\%; m.p. $152-155{ }^{\circ} \mathrm{C}$ Anal. Calc. for $\mathrm{C}_{13} \mathrm{H}_{11} \mathrm{NO}_{3}(229.24) \mathrm{C}, 68.11 ; \mathrm{H}, 4.84 ; \mathrm{N}, 6.11$. Found: $\mathrm{C}, 68.29 ; \mathrm{H}, 5.06 ; \mathrm{N}, 6.14 \%$. IR: $1627.7(\mathrm{v} / \mathrm{C}=\mathrm{N})$, ${ }^{1} \mathrm{H}-\mathrm{NMR}\left(\mathrm{DMSO}-d_{6}\right) \delta: 14.20(\mathrm{~s}, 1 \mathrm{H}), 10.13(\mathrm{~s}, 1 \mathrm{H}), 9.62(\mathrm{~s}, 1 \mathrm{H}), 8.76(\mathrm{~s}, 1 \mathrm{H}), 7.35(\mathrm{~d}, J=8.6 \mathrm{~Hz}, 1 \mathrm{H}), 7.28$ $(\mathrm{dd}, J=7.9,1.5 \mathrm{~Hz}, 1 \mathrm{H}), 7.10-7.01(\mathrm{~m}, 1 \mathrm{H}), 6.91(\mathrm{dd}, J=8.0,1.3 \mathrm{~Hz}, 1 \mathrm{H}), 6.83(\mathrm{td}, J=7.8,1.4 \mathrm{~Hz}, 1 \mathrm{H})$, $6.32(\mathrm{dd}, J=8.5,2.3 \mathrm{~Hz}, 1 \mathrm{H}), 6.21(\mathrm{~d}, J=2.3 \mathrm{~Hz}, 1 \mathrm{H}) .{ }^{13} \mathrm{C}-\mathrm{NMR}\left(\mathrm{DMSO}-d_{6}\right) \delta: 165.40,162.90,160.66$, $150.90,134.91,134.62,127.52,120.03,119.47,116.75,112.66,107.94,103.09$; positive LC-MS $m / z: 230.0$ $[\mathrm{M}+\mathrm{H}]^{+}$calc. for $\mathrm{C}_{13} \mathrm{H}_{12} \mathrm{NO}_{3}{ }^{+}, 230.08$, found 230.0.

2-\{[(2-Hydroxyphenyl)imino]methyl\}benzene-1,4-diol (10): Yield 71\%; m.p. $135-137^{\circ} \mathrm{C}$. Anal. Calc. for $\mathrm{C}_{13} \mathrm{H}_{11} \mathrm{NO}_{3}$ (229.24) C, 68.11; H, 4.84; N, 6.11. Found: C, 67.92; H, 4.72; N, 5.88 \%. IR: $1638.5(v / \mathrm{C}=\mathrm{N})$, ${ }^{1} \mathrm{H}-\mathrm{NMR}\left(\mathrm{DMSO}-d_{6}\right) \delta: 12.86(\mathrm{~s}, 1 \mathrm{H}), 9.63(\mathrm{~s}, 1 \mathrm{H}), 9.03(\mathrm{~s}, 1 \mathrm{H}), 8.83(\mathrm{~s}, 1 \mathrm{H}), 7.32(\mathrm{dd}, J=7.9,1.5 \mathrm{~Hz}$, 1H), 7.16-7.05 (m, 1H), 7.03-6.72 (m, 5H). ${ }^{13} \mathrm{C}-\mathrm{NMR}$ (DMSO-d 6 ) $\delta: 162.02,153.79,151.48,149.80,135.91$, $128.28,121.09,120.05,120.01,119.90,117.56,117.31,116.90$; positive LC-MS $m / z: 230.1[\mathrm{M}+\mathrm{H}]^{+}$calc. for $\mathrm{C}_{13} \mathrm{H}_{12} \mathrm{NO}_{3}{ }^{+}, 230.08$, found 230.1 .

5-\{[(2-Hydroxyphenyl)imino]methyl\}benzene-1,3-diol (11): Yield 26\%; m.p. $174-175{ }^{\circ} \mathrm{C}$. Anal. Calc. for $\mathrm{C}_{13} \mathrm{H}_{11} \mathrm{NO}_{3}$ (229.24) C, 68.11; H, 4.84; N, 6.11. Found: C, 68.32; H, 4.69; N, 5.86\%. IR: $1628.1(\mathrm{v} / \mathrm{C}=\mathrm{N})$. ${ }^{1} \mathrm{H}-\mathrm{NMR}\left(\mathrm{DMSO}-d_{6}\right): \delta=9.45(\mathrm{~s}, 2 \mathrm{H}), 8.98(\mathrm{~s}, 1 \mathrm{H}), 8.44(\mathrm{~s}, 1 \mathrm{H}), 7.10(\mathrm{dd}, J=7.8,1.5 \mathrm{~Hz}, 1 \mathrm{H}), 7.05(\mathrm{ddd}$, $J=7.8,7.7,1.5,1 \mathrm{H}), 6.87(\mathrm{dd}, \mathrm{J}=8.0,1.3 \mathrm{~Hz}, 1 \mathrm{H}), 6.85(\mathrm{~d}, J=2.2 \mathrm{~Hz}, 2 \mathrm{H}), 6.81(\mathrm{ddd}, J=7.7,7.4,1.3 \mathrm{~Hz}$, $1 \mathrm{H}), 6.36(\mathrm{dd}, J=2.2 \mathrm{~Hz}, 1 \mathrm{H}) .{ }^{13} \mathrm{C}-\mathrm{NMR}\left(\mathrm{DMSO}-d_{6}\right): \delta=160.40,158.97,151.18,138.87,138.64,127.40$, $119.98,119.88,116.35,107.30,105.99$; positive LC-MS $m / z: 230.1[\mathrm{M}+\mathrm{H}]^{+}$calc. for $\mathrm{C}_{13} \mathrm{H}_{12} \mathrm{NO}_{3}{ }^{+}, 230.08$, found 230.1 .

3-\{[(3-Hydroxyphenyl)imino]methyl\}benzene-1,2-diol (12): Yield 79\%; m.p. $184-185{ }^{\circ} \mathrm{C}$. Anal. Calc. for $\mathrm{C}_{13} \mathrm{H}_{11} \mathrm{NO}_{3}$ (229.24) C, 68.11; H, 4.84; N, 6.11. Found: C, 68.32; H, 4.65; N, 5.87\%. IR: $1632.9(v / \mathrm{C}=\mathrm{N})$, ${ }^{1} \mathrm{H}-\mathrm{NMR}\left(\mathrm{DMSO}-d_{6}\right) \delta: 13.25(\mathrm{~s}, 1 \mathrm{H}), 9.64(\mathrm{~s}, 1 \mathrm{H}), 9.18(\mathrm{~s}, 1 \mathrm{H}), 8.86(\mathrm{~s}, 1 \mathrm{H}), 7.25(\mathrm{dd}, J=7.9 \mathrm{~Hz}, 1 \mathrm{H})$, $7.09(\mathrm{dd}, J=7.8,1.4 \mathrm{~Hz}, 1 \mathrm{H}), 6.94(\mathrm{dd}, J=7.8,1.4 \mathrm{~Hz}, 1 \mathrm{H}), 6.88-6.67(\mathrm{~m}, 4 \mathrm{H}) .{ }^{13} \mathrm{C}-\mathrm{NMR}\left(\mathrm{DMSO}-d_{6}\right)$ 
$\delta: 163.57,158.34,149.48,149.01,145.62,130.24,122.81,119.28,118.91,118.72,114.07,111.97,108.13$; positive LC-MS m/z: $230.1[\mathrm{M}+\mathrm{H}]^{+}$calc. for $\mathrm{C}_{13} \mathrm{H}_{12} \mathrm{NO}_{3}{ }^{+}, 230.08$, found 230.1.

2-\{[(3-Hydroxyphenyl)imino]methyl\}benzene-1,4-diol (13): Yield 89\%; m.p. $175-177^{\circ} \mathrm{C}$. Anal. Calc. for $\mathrm{C}_{13} \mathrm{H}_{11} \mathrm{NO}_{3}$ (229.24) C, 68.11; H, 4.84; N, 6.11. Found: 68.30; H, 4.73; N, 5.91\%. IR: $1634.1(\mathrm{v} / \mathrm{C}=\mathrm{N})$, ${ }^{1} \mathrm{H}-\mathrm{NMR}\left(\mathrm{DMSO}-d_{6}\right) \delta: 12.27(\mathrm{~s}, 1 \mathrm{H}), 9.62(\mathrm{~s}, 1 \mathrm{H}), 9.08(\mathrm{~s}, 1 \mathrm{H}), 8.78(\mathrm{~s}, 1 \mathrm{H}), 7.23(\mathrm{dd}, J=7.9 \mathrm{~Hz}, 1 \mathrm{H})$, $7.03(\mathrm{~d}, J=2.9 \mathrm{~Hz}, 1 \mathrm{H}), 6.91-6.64(\mathrm{~m}, 5 \mathrm{H}) .{ }^{13} \mathrm{C}-\mathrm{NMR}\left(\mathrm{DMSO}-d_{6}\right) \delta: 163.29,158.70,153.50,150.11,150.02$, $130.56,121.47,119.65,117.55,117.38,114.32,112.46,108.53$; positive LC-MS $m / z: 230.1[\mathrm{M}+\mathrm{H}]^{+}$calc. for $\mathrm{C}_{13} \mathrm{H}_{12} \mathrm{NO}_{3}{ }^{+}, 230.08$, found 230.1 .

4-\{[(4-Hydroxyphenyl)imino]methyl\}benzene-1,3-diol (14): Yield 55\%; m.p. $130-132{ }^{\circ} \mathrm{C}$. Anal. Calc. for $\mathrm{C}_{13} \mathrm{H}_{11} \mathrm{NO}_{3}$ (229.24) C, 68.11; H, 4.84; N, 6.11. Found: 68.27; H, 5.06; N, 5.90\%. IR: $1617.4(\mathrm{v} / \mathrm{C}=\mathrm{N})$. ${ }^{1} \mathrm{H}-\mathrm{NMR}\left(\mathrm{DMSO}-d_{6}\right) \delta: 13.80(\mathrm{~s}, 1 \mathrm{H}), 10.13(\mathrm{~s}, 1 \mathrm{H}), 9.55(\mathrm{~s}, 1 \mathrm{H}), 8.72(\mathrm{~s}, 1 \mathrm{H}), 7.37(\mathrm{~d}, J=8.5 \mathrm{~Hz}, 1 \mathrm{H})$, 7.29-7.16 (m, 2H), 6.86-6.76 (m, 2H), $6.38(\mathrm{dd}, J=8.4,2.3 \mathrm{~Hz}, 1 \mathrm{H}), 6.27(\mathrm{~d}, J=2.3 \mathrm{~Hz}, 1 \mathrm{H}) .{ }^{13} \mathrm{C}-\mathrm{NMR}$ (DMSO-d $d_{6}$ ) $\delta: ~ 163.19,162.23,160.18,156.67,139.86,134.33,122.57,116.31,112.59,107.98,102.79$; positive LC-MS m/z: $230.1[\mathrm{M}+\mathrm{H}]^{+}$calc. for $\mathrm{C}_{13} \mathrm{H}_{12} \mathrm{NO}_{3}{ }^{+}, 230.08$, found 230.1 .

2-\{[(4-Hydroxyphenyl)imino]methyl\}benzene-1,4-diol (15): Yield 92\%; m.p. 196-197 ${ }^{\circ} \mathrm{C}$. Anal. Calc. for $\mathrm{C}_{13} \mathrm{H}_{11} \mathrm{NO}_{3}$ (229.24) C, 68.11; H, 4.84; N, 6.11. Found: 67.92; H, 4.97; N, 6.26\%. IR: $1624.5(v / \mathrm{C}=\mathrm{N})$. ${ }^{1} \mathrm{H}-\mathrm{NMR}\left(\mathrm{DMSO}_{-} d_{6}\right) \delta: 12.58(\mathrm{~s}, 1 \mathrm{H}), 9.64(\mathrm{~s}, 1 \mathrm{H}), 9.04(\mathrm{~s}, 1 \mathrm{H}), 8.79(\mathrm{~s}, 1 \mathrm{H}), 7.29(\mathrm{~d}, J=8.7 \mathrm{~Hz}, 2 \mathrm{H}), 6.98$ $(\mathrm{d}, J=2.7 \mathrm{~Hz}, 1 \mathrm{H}), 6.93-6.52(\mathrm{~m}, 4 \mathrm{H}) .{ }^{13} \mathrm{C}-\mathrm{NMR}\left(\mathrm{DMSO}-d_{6}\right) \delta: 160.39,157.26,153.35,149.94,139.99$, 123.05, 120.70, 119.82, 117.40, 117.27, 116.35; positive LC-MS $m / z: 230.1[\mathrm{M}+\mathrm{H}]^{+}$calc. for $\mathrm{C}_{13} \mathrm{H}_{12} \mathrm{NO}_{3}{ }^{+}$, 230.08, found 230.1.

5-\{[(4-Hydroxyphenyl)imino]methyl\}benzene-1,3-diol (16): Yield 92\%; m.p. $118-120{ }^{\circ} \mathrm{C}$. Anal. Calc. for $\mathrm{C}_{13} \mathrm{H}_{11} \mathrm{NO}_{3}(229.24) \mathrm{C}, 68.11 ; \mathrm{H}, 4.84 ; \mathrm{N}, 6.11$. Found: 67.92; H, 4.97; N, 6.26\%. IR: $1620.5(v / \mathrm{C}=\mathrm{N})$. ${ }^{1} \mathrm{H}-\mathrm{NMR}\left(\mathrm{DMSO}-d_{6}\right) \delta: 9.44(\mathrm{~s}, 3 \mathrm{H}), 8.38(\mathrm{~s}, 1 \mathrm{H}), 7.15(\mathrm{~d}, J=8.7 \mathrm{~Hz}, 2 \mathrm{H}), 6.87-6.69(\mathrm{~m}, 4 \mathrm{H}), 6.32$ $(\mathrm{dd}, J=2.1 \mathrm{~Hz}, 1 \mathrm{H}) .{ }^{13} \mathrm{C}-\mathrm{NMR}$ (DMSO- $\left.d_{6}\right) \delta: 159.05,157.85,156.61,143.02,138.78,122.90,116.12$, 106.80, 105.66; positive LC-MS $m / z: 230.1[\mathrm{M}+\mathrm{H}]^{+}$calc. for $\mathrm{C}_{13} \mathrm{H}_{12} \mathrm{NO}_{3}{ }^{+}, 230.08$, found 230.1.

4-\{[(3-Hydroxyphenyl)imino]methyl\}benzene-1,2,3-triol (17): Yield 89\%; m.p. $254-256{ }^{\circ} \mathrm{C}$. Anal. Calc. for $\mathrm{C}_{13} \mathrm{H}_{11} \mathrm{NO}_{4}(245.24) \mathrm{C}, 63.67 ; \mathrm{H}, 4.52 ; \mathrm{N}, 5.71$. Found: 63.96; H, 4.48; N, 5.50\%. IR: $1606.2(v / \mathrm{C}=\mathrm{N})$. ${ }^{1} \mathrm{H}-\mathrm{NMR}\left(\mathrm{DMSO}-d_{6}\right) \delta: 13.70(\mathrm{~s}, 1 \mathrm{H}), 9.63(\mathrm{~d}, 2 \mathrm{H}), 8.72(\mathrm{~s}, 1 \mathrm{H}), 8.43(\mathrm{~s}, 1 \mathrm{H}), 7.22(\mathrm{dd}, J=7.9 \mathrm{~Hz}, 1 \mathrm{H})$, $6.94(\mathrm{~d}, J=8.6 \mathrm{~Hz}, 1 \mathrm{H}), 6.78(\mathrm{~d}, J=8.9 \mathrm{~Hz}, 1 \mathrm{H}), 6.76-6.62(\mathrm{~m}, 2 \mathrm{H}), 6.40(\mathrm{~d}, J=8.5 \mathrm{~Hz}, 1 \mathrm{H}) .{ }^{13} \mathrm{C}-\mathrm{NMR}$ (DMSO- $d_{6}$ ) $\delta:$ 163.25, 158.96, 152.61, 150.89, 149.38, 133.05, 130.83, 124.74, 114.04, 112.82, 112.23, 108.41; positive LC-MS m/z: $246.1[\mathrm{M}+\mathrm{H}]^{+}, 268.0[\mathrm{M}+\mathrm{Na}]^{+}$. Calc. for $\mathrm{C}_{13} \mathrm{H}_{12} \mathrm{NO}_{3}{ }^{+}, 246.08$, found 246.1.

2-\{[(3-Hydroxyphenyl)imino]methyl\}benzene-1,3,5-triol (18): Yield 94\%; m.p. 257-259 ${ }^{\circ} \mathrm{C}$. Anal. Calc. for $\mathrm{C}_{13} \mathrm{H}_{11} \mathrm{NO}_{4}$ (245.24) C, 63.67; H, 4.52; N, 5.71. Found: 63.87; H, 4.39; N, 5.78\%. IR: $1626.1(v / \mathrm{C}=\mathrm{N})$. ${ }^{1} \mathrm{H}-\mathrm{NMR}\left(\mathrm{DMSO}-d_{6}\right) \delta: 12.25(\mathrm{~s}, 2 \mathrm{H}), 10.07(\mathrm{~s}, 1 \mathrm{H}), 9.56(\mathrm{~s}, 1 \mathrm{H}), 8.87(\mathrm{~s}, 1 \mathrm{H}), 7.20(\mathrm{dd}, J=11.1,5.5 \mathrm{~Hz}$, $1 \mathrm{H}), 6.68(\mathrm{dd}, J=18.1,7.3 \mathrm{~Hz}, 3 \mathrm{H}), 5.81(\mathrm{~s}, 2 \mathrm{H}) .{ }^{13} \mathrm{C}-\mathrm{NMR}$ (DMSO- $\left.d_{6}\right) \delta: 163.91,162.62,158.33,157.10$, 149.27, 130.28, 113.11, 111.57, 107.33, 101.46, 94.10; positive LC-MS $m / z: 246.1[\mathrm{M}+\mathrm{H}]^{+}$, calc. for $\mathrm{C}_{13} \mathrm{H}_{12} \mathrm{NO}_{3}{ }^{+}, 246.08$, found 246.1 .

5-\{[(3-Hydroxyphenyl)imino]methyl\}benzene-1,2,3-triol (19): Yield 47\%; m.p. 283-285 ${ }^{\circ} \mathrm{C}$. Anal. Calc. for $\mathrm{C}_{13} \mathrm{H}_{11} \mathrm{NO}_{4}$ (245.24) C, 63.67; H, 4.52; N, 5.71. Found: 63.77; H, 4.39; N, 5.78\%. IR: $1644.2(v / \mathrm{C}=\mathrm{N})$. ${ }^{1} \mathrm{H}-\mathrm{NMR}$ (DMSO- $d_{6}$ ) $\delta: 9.42(\mathrm{~s}, 1 \mathrm{H}), 9.17(\mathrm{~s}, 2 \mathrm{H}), 8.76(\mathrm{~s}, 1 \mathrm{H}), 8.22(\mathrm{~s}, 1 \mathrm{H}), 7.14(\mathrm{dd}, J=7.9 \mathrm{~Hz}, 1 \mathrm{H}), 6.87$ $(\mathrm{s}, 2 \mathrm{H}), 6.70-6.48(\mathrm{~m}, 3 \mathrm{H}) \mathrm{m} .{ }^{13} \mathrm{C}-\mathrm{NMR}$ (DMSO-d $\left.{ }_{6}\right) \delta: 192.00,160.66,158.66,153.92,150.43,146.78,146.68$, 140.86, 137.71, 130.43, 130.07, 128.10, 127.40, 112.97, 112.21, 109.41, 108.56, 108.35, 106.07, 103.94, 101.60; positive LC-MS m/z: $246.1[\mathrm{M}+\mathrm{H}]^{+}, 268.1[\mathrm{M}+\mathrm{Na}]^{+}$. Calc. for $\mathrm{C}_{13} \mathrm{H}_{12} \mathrm{NO}_{3}{ }^{+}, 246.08$, found 246.1 .

2-\{[(4-Hydroxyphenyl)imino]methyl\}benzene-1,3,5-triol (20): Yield 37\%; m.p. 173-175 ${ }^{\circ} \mathrm{C}$. Anal. Calc. for $\mathrm{C}_{13} \mathrm{H}_{11} \mathrm{NO}_{4}$ (245.24) C, 63.67; H, 4.52; N, 5.71. Found: C, 63.76; H, 4.69; N, 5.88\%. IR: $1616.0(\mathrm{v} / \mathrm{C}=\mathrm{N})$. ${ }^{1} \mathrm{H}-\mathrm{NMR}\left(\mathrm{DMSO}_{-} d_{6}\right) \delta: 12.27(\mathrm{~s}, 2 \mathrm{H}), 9.94(\mathrm{~s}, 1 \mathrm{H}), 9.48(\mathrm{~s}, 1 \mathrm{H}), 8.85(\mathrm{~s}, 1 \mathrm{H}), 7.14(\mathrm{~d}, J=8.7 \mathrm{~Hz}, 2 \mathrm{H}), 6.79$ 
$(\mathrm{d}, J=8.7 \mathrm{~Hz}, 2 \mathrm{H}), 5.79(\mathrm{~s}, 2 \mathrm{H}) .{ }^{13} \mathrm{C}-\mathrm{NMR}\left(\mathrm{DMSO}-d_{6}\right) \delta: 163.53,156.34,155.39,140.10,122.21,116.39$, 101.85, 94.44; positive LC-MS m/z: $246.1[\mathrm{M}+\mathrm{H}]^{+}$, calc. for $\mathrm{C}_{13} \mathrm{H}_{12} \mathrm{NO}_{3}{ }^{+}, 246.08$, found 246.1 .

5-\{[(4-Hydroxyphenyl)imino]methyl\}benzene-1,2,3-triol (21): Yield 89\%; m.p. 228-229 ${ }^{\circ} \mathrm{C}$. Anal. Calc. for $\mathrm{C}_{13} \mathrm{H}_{11} \mathrm{NO}_{4}$ (245.24) C, 63.67; H, 4.52; N, 5.71. Found: C, 63.80; H, 4.41; N, 5.87\%. IR: $1640.0(\mathrm{v} / \mathrm{C}=\mathrm{N})$. ${ }^{1} \mathrm{H}-\mathrm{NMR}\left(\mathrm{DMSO}-d_{6}\right) \delta: 9.35(\mathrm{~s}, 1 \mathrm{H}), 9.11(\mathrm{~s}, 2 \mathrm{H}), 8.59(\mathrm{~d}, 1 \mathrm{H}), 8.26(\mathrm{~s}, 1 \mathrm{H}), 7.23-7.00(\mathrm{~m}, 2 \mathrm{H}), 6.85$ $(\mathrm{d}, J=2.8 \mathrm{~Hz}, 2 \mathrm{H}), 6.81-6.67(\mathrm{~m}, 2 \mathrm{H}) .{ }^{13} \mathrm{C}-\mathrm{NMR}\left(\mathrm{DMSO}-d_{6}\right) \delta: 168.82,148.74,145.74,140.95,136.83$, 124.97, 116.02, 115.85, 107.57; positive LC-MS $m / z: 246.1[\mathrm{M}+\mathrm{H}]^{+}, 268.0[\mathrm{M}+\mathrm{Na}]^{+}$. Calc. for $\mathrm{C}_{13} \mathrm{H}_{12} \mathrm{NO}_{3}{ }^{+}, 246.08$, found 246.1 .

\subsection{DPPH Assay}

Scavenging of DPPH radicals by prepared (hydroxyphenyliminomethyl)phenols was performed according to our previous work Šeršeň et al. [34]. Briefly, various amounts of tested (Hydroxy phenyliminomethyl)phenols were added into methanol solution of DPPH and the final DPPH concentration was kept constant $\left(c=10^{-4} \mathrm{~mol} \cdot \mathrm{dm}^{-3}\right) .20 \mathrm{~min}$ after the addition of the tested substance, absorbance was measured at $517 \mathrm{~nm}$. The antioxidant activity was evaluated using the values $\mathrm{SC}_{50}$, i.e., concentration of the studied compound, which causes a $50 \%$ decrease in absorbance at $517 \mathrm{~nm}$ as compared to the control sample. Methanol was used as a blank.

\subsection{Scavenging of Galvinoxyl Radicals}

Various amounts of (hydroxyphenyliminomethyl)phenols were added into methanol solution of galvinoxyl radical such that the final galvinoxyl concentration was $c=10^{-4} \mathrm{~mol} \cdot \mathrm{dm}^{-3} .20 \mathrm{~min}$ after the addition of (hydroxyphenyliminomethyl)phenols, absorbance was measured at $862 \mathrm{~nm} . \mathrm{SC}_{50}$ values were calculated from the absorbance $v$ s. the concentration dependence. Methanol was used as a blank.

\subsection{ABTS Assay}

ABTS cation radicals were prepared according to Kurin et al. [35] with minor adaptations. Briefly $7 \mathrm{mM}$ aqueous solution of ABTS was mixed in equimolar ratio with $2.45 \mathrm{mM} \mathrm{K} \mathrm{S}_{2} \mathrm{O}_{8}$, and let $24 \mathrm{~h}$ to stand in the dark at room temperature. After $24 \mathrm{~h} 1.1 \mathrm{~mL}$ of concentrated solution of the radical was diluted with $50 \mathrm{~mL}$ of phosphate buffer (solution A: $900 \mathrm{mg}$ of $\mathrm{Na}_{2} \mathrm{HPO}_{4}$ in $500 \mathrm{~mL} \mathrm{H}_{2} \mathrm{O}$, the solution B: $340 \mathrm{mg}$ of $\mathrm{KH}_{2} \mathrm{HPO}_{4}$ in $500 \mathrm{~mL} \mathrm{H} \mathrm{H}_{2} \mathrm{O}$, we combined 9 parts of solution A and 1 part of solution $\mathrm{B}$, the $\mathrm{pH}$ we set to 7.4). To a solution of $1.8 \mathrm{~mL}$ of ABTS we added $0,25,50,75,100,125,150$, 175 or $200 \mathrm{~mL}$ of the test substance and completed with 200,175, 150, 125, 100, 75, 50, 25 or $0 \mathrm{~mL}$ of ethanol (to a final volume of $2 \mathrm{~mL}$. The absorbance at $734 \mathrm{~nm}$ of prepared solutions was measured after $6 \mathrm{~min}$. We calculated the appropriate values of the $\mathrm{SC}_{50}$ from the linear part of the absorbance $v$ s. the concentration of (hydroxyphenyliminomethyl)phenols. The water was used as blank.

\subsection{Molecular Calculations}

The prepared (hydroxyphenyliminomethyl)phenols, their anions and radicals were studied using the quantum chemical method PM6 [36], which is part of the program MOPAC2012 [37]. Optimal structures of compunds were calculated (keyword PRECISE). The effect of solvents on the above mentioned compounds were studied by COSMO-method [38], which is also part of the MOPAC2012 [39]. Ionization potentials and heats of formations used for the calculation of PDE, BDE, PA and ETE according to the Equations (2), (6), (10) and (11) were obtained using the method PM6.

\section{Conclusions}

In this work we prepared twenty-one (hydroxyphenyliminomethyl)phenols, which were synthesized by condensation reactions of the corresponding aminophenols and hydroxybenzaldehydes. The chemical structures of these compounds were confirmed by ${ }^{1} \mathrm{H}-\mathrm{NMR}$, IR spectroscopy, elemental analysis and GCMS. All prepared (hydroxyphenyliminomethyl)phenols were able to 
scavenge DPPH, GOR and ABTS radicals. Some of the (hydroxyphenyliminomethyl)phenols were more potent scavengers of DPPH (compounds: 8, 10, 12-14, 11-27) and GOR (compounds: $\mathbf{2}, \mathbf{9}, \mathbf{1 2}, \mathbf{1 5}, \mathbf{1 7}-\mathbf{2 1}$ ) radicals as compared to resveratrol. The efficiency of antioxidant activity correlated with the number and position of hydroxyl groups. The most efficient antioxidants were (hydroxyphenyliminomethyl)phenols containing three hydroxyl groups in the benzylidene part of molecules.

Supplementary Materials: Supplementary materials can be accessed at: http://www.mdpi.com/1420-3049/ $21 / 1 / 127 / \mathrm{s} 1$.

Acknowledgments: This work was supported by the Slovak Grant Agency VEGA 1/0612/11 and 1/0196/14, projects ITMS 26240220071, APVV-0334-12, APVV-0061-11, PCIG11-GA-2012-322300 and The Austrian Science Fund (FWF) grants P23609 and P21437. We are greatful to Julia Greganova for her help.

Author Contributions: F.G., F.Š., P.K. and J.F. performed experiments. D.L. performed calculations. F.Š., F.G. and J.G. analysed results and wrote the manuscript. All authors contributed to the paper and approved the manuscript.

Conflicts of Interest: The authors declare no conflict of interest.

\section{Abbreviations}

The following abbreviations are used in this manuscript:

$\begin{array}{ll}\text { ABTS } & \text { 2,2'-azino-bis(3-ethylbenzothiazoline)-6-sulphonic acid } \\ \text { AP } & \text { aminophenol } \\ \text { BDE } & \text { bond dissociation enthalpy } \\ \text { DPPH } & \text { 2,2-diphenyl-1-picrylhydrazyl } \\ \text { ETE } & \text { electron transfer enthalpy } \\ \text { GOR } & \text { galvinoxyl radical } \\ \text { HBA } & \text { hydroxybenzaldehyde } \\ \text { IP } & \text { ionization potential } \\ \text { IR } & \text { infrared } \\ \text { MS } & \text { mass spectrometry } \\ \text { NMR } & \text { nuclear magnetic resonance } \\ \text { PA } & \text { proton affinity }\end{array}$

\section{References}

1. Celotti, E.; Ferrarini, R.; Zironi, R.; Conte, L.S. Resveratrol content of some wines obtained from dried Valpolicella grapes: Recioto and Amarone. J. Chromatogr. A 1996, 730, 47-52. [CrossRef]

2. Soleas, G.J.; Diamandis, E.P.; Goldberg, D.M. Resveratrol: A molecule whose time has come? And gone? Clin. Biochem. 1997, 30, 91-113. [CrossRef]

3. Gu, X.L.; Chub, Q.Y.; O'Dwyer, M.; Zeece, M. Analysis of resveratrol in wine by capillary electrophoresis. J. Chromatogr. A 2000, 881, 471-481. [CrossRef]

4. Kaeberlein, K.; McDonagh, T.; Heltweg, B.; Hixon, J.; Westman, E.A.; Caldwell, S.D.; Napper, A.; Curtis, R.; DiStefano, P.S.; Fields, S.; et al. Substrate-specific activation of sirtuins by resveratrol. J. Biol. Chem. 2005, 280, 17038-17045. [CrossRef] [PubMed]

5. Renaud, S.; de Lorgeril, M. Wine, alcohol, platelets, and the French paradox for coronary heart disease. Lancet 1992, 339, 1523-1526. [CrossRef]

6. Santos, J.A.; de Carvaho, G.S.; Oliveira, V.; Raposo, N.R.; da Silva, A.D. Resveratrol and analogues: A review of antioxidant activity and applications to human health. Recent Pat. Food Nutr. Agric. 2013, 5, 144-153. [CrossRef] [PubMed]

7. Yan, L.J. Positive oxidative stress in aging and aging-related disease tolerance. Redox Biol. 2014, 2C, 165-169. [CrossRef] [PubMed] 
8. Ghanim, H.; Sia, C.L.; Korzeniewski, K.; Lohano, T.; Abuaysheh, S.; Marumganti, A.; Chaudhuri, A.; Dandona, P. A resveratrol and polyphenol preparation suppresses oxidative and inflammatory stress response to a high-fat, high-carbohydrate meal. J. Clin. Endocrinol. Metab. 2011, 96, 1409-1414.

9. Hubbard, B.P.; Sinclair, D.A. Small molecule SIRT1 activators for the treatment of aging and age-related diseases. Trends Pharmacol. Sci. 2014, 35, 146-154. [CrossRef] [PubMed]

10. Li, C.; Xu, X.; Wang, X.J.; Pan, Y. Imine resveratrol analogues: Molecular design, NRF2 activation and SAR analysis. PLoS ONE 2014, 9, e101455.

11. Franco, D.C.Z.; de Carvalho, G.S.G.; Rocha, P.R.; da Silva Teixeira, R.; da Silva, A.D.; Raposo, N.R.B. Inhibitory effects of resveratrol analogs on mushroom tyrosinase activity. Molecules 2012, 17, 11816-11825. [CrossRef] [PubMed]

12. Chalal, M.; Klinguer, A.; Echairi, A.; Meunier, P.; Vervandier-Fasseur, D.; Adrian, M. Antimicrobial activity of resveratrol analogues. Molecules 2014, 19, 7679-7688. [CrossRef] [PubMed]

13. Alexander, V. Design and synthesis of macrocyclic ligands and their complexes of lanthanides and actinides. Chem. Rev. 1995, 95, 273-342. [CrossRef]

14. Westheimer, F.H.; Taguchi, K. Catalysis by molecular sieves in the preparation of ketimines and enamines. J. Org. Chem. 1971, 36, 1570-1572. [CrossRef]

15. Castellano, J.A.; Goldmacher, J.E.; Barton, L.A.; Kane, J.S. Liquid crystals. II. Effects of terminal group substitution on the mesomorphic behavior of some benzylideneanilines. J. Org. Chem. 1968, 33, 3501-3504. [CrossRef]

16. Tanaka, K.; Shiraishi, R. Clean and efficient condensation reactions of aldehydes and amines in a water suspension medium. Green Chem. 2000, 2, 272-273. [CrossRef]

17. Van den Ancker, T.R.; Cave, G.W.V.; Raston, C.L. Benign approaches for the synthesis of bis-imine Schiff bases. Green Chem. 2006, 8, 50-53. [CrossRef]

18. Koldas, S.; Demitras, I.; Ozen, T.; Demirici, M.; Behcet, L. Phytochemical screening, anticancer and antioxidant activities of Origanum vulgare L. ssp viride (Boiss.) Hayek, a plant of traditional usage. J. Sci. Food Agric. 2015, 95, 786-798. [CrossRef] [PubMed]

19. Kawamura, F.; Saary, N.S.; Hashim, R.; Sulaiman, O.; Hashida, K.; Otsuka, Y.; Nakamura, M.; Ohara, S. Subcritical water extraction of low-molecular-weight phenolic compounds from oil palm biomass. Jpn. Agr. Res. Q. 2014, 48, 355-362. [CrossRef]

20. Choudhary, A.; Mittal, A.K.; Radhika, M.; Tripathy, D.; Chatterjee, A.; Banerjee, U.C.; Singh, I.P. Two new stereoisomeric antioxidant triterpenes from Potentilla fulgens. Fitoterapia 2013, 91, 290-297.

21. Asikin, Y.; Takahashi, M.; Mishima, T.; Mizu, M.; Takara, K.; Wada, K. Antioxidant activity of sugarcane molasses against 2,2'-azobis(2-amidinopropane) dihydrochloride-induced peroxyl radicals. Food Chem. 2013, 141, 466-472. [CrossRef] [PubMed]

22. Rodríguez-Jimenes, G.C.; Vargas-Garcia, A.; Espinoza-Pérez, D.J.; Salgado-Cervantes, M.A.; Robles-Olvera, V.J. García-Alvarado, M.A. Mass transfer during vanilla pods solid liquid extraction: Effect of extraction method. Food Bioprocess Technol. 2013, 6, 2640-2650. [CrossRef]

23. Razafintsalama, V.; Sarter, S.; Mambu, L.; Randrianarivo, R.; Petit, T.; Rajaonarison, J.F.; Mertz, C.; Rakoto, D.; Jeannoda, V. Antimicrobial activities of Dilobeia thouarsii Roemer and Schulte, a traditional medicinal plant from Madagascar. S. Afr. J. Bot. 2013, 87, 1-3. [CrossRef]

24. Friedman, M.; Henika, P.R.; Mandrell, R.E. Antibacterial Activities of phenolic benzaldehydes and benzoic acids against Campylobacter jejuni, Escherichia coli, Listeria monocytogenes, and Salmonella enterica. J. Food Prot. 2003, 66, 1811-1821. [PubMed]

25. Mangas, J.; Rodriguez, R.; Moreno, J.; Suarez, B.; Blanco, D. Evolution of aromatic and furanic congeners in the maturation of cider brandy: A contribution to its characterization. J. Agric. Food Chem. 1996, 44, 3303-3307. [CrossRef]

26. Bountagkidou, O.G.; Ordoudi, S.A.; Tsimidou, M.Z. Structure-antioxidant activity relationship study of natural hydroxybenzaldehydes using in vitro assays. Food Res. Int. 2010, 43, 2014-2019. [CrossRef]

27. Nenadis, N.; Tsimidou, M.Z. Contribution of DFT computed molecular descriptors in the study of radical scavenging activity trend of natural hydroxybenzaldehydes and corresponding acids. Food Res. Int. 2012, 48, 538-543. [CrossRef]

28. Li, S.Y.; Wang, X.B.; Kong, L.Y. Design, synthesis and biological evaluation of imine resveratrol derivatives as multi-targeted agents against Alzheimer's disease. Eur. J. Med. Chem. 2014, 71, 36-45. [CrossRef] [PubMed] 
29. Lu, J.; Li, C.; Chai, Y.F.; Yang, D.Y.; Sun, C.R. The antioxidant effect of imine resveratrol analogues. Bioorg. Med. Chem. Lett. 2012, 22, 5744-5747. [CrossRef] [PubMed]

30. Šeršeň, F.; Walko, M.; Loos, D. Antioxidative effect of some hydroxy substituted aromatic bisimines. Gen. Physiol. Biophys. 2009, 28, 210-214. [CrossRef] [PubMed]

31. Sharma, V.; Khan, M.S.Y. Synthesis of novel tetrahydroimidazole derivatives and studies for their biological properties. Eur. J. Med. Chem. 2001, 36, 651-658. [CrossRef]

32. Cigáň, M.; Jakusová, K.; Donovalová, J.; Filo, J.; Horváth, M.; Gáplovský, A. Fluorescence of isatin $\mathrm{N}$-phenylsemicarbazones: Aggregation and hydrazide-hydrazonol tautomerism. J. Phys. Org. Chem. 2015, 28, 337-346. [CrossRef]

33. Gobec, M.; Tomašič, T.; Markovič, T.; Mlinarič-Raščan, I.; Dolenc, M.S.; Jakopin, Ž. Antioxidant and anti-inflammatory properties of 1,2,4-oxadiazole analogs of resveratrol. Chem. Biol. Interact. 2015, 240, 200-207. [CrossRef] [PubMed]

34. Šeršeň, F.; Mučaji, P.; Grančai, D.; Nagy, M. Antioxidative properties of methanol extracts from leaves and fruits of Ligustrum vulgare L. Acta Facult. Pharm. Univ. Comen. 2005, 52, 204-209.

35. Kurin, E.; Mučaji, P.; Nagy, M. In vitro antioxidant activities of three red wine polyphenols and their mixtures: An interaction study. Molecules 2012, 17, 14336-14348. [CrossRef] [PubMed]

36. Stewart, J.J.P. Optimization of parameters for semiempirical methods V: Modification of NDDO approximations and application to 70 elements. J. Mol. Model. 2007, 13, 1173-1213. [CrossRef] [PubMed]

37. MOPAC, version 2012; Stewart Computational Chemistry: Colorado Springs, CO, USA, 2012.

38. Klamt, A.; Schüümann, G. COSMO: A new approach to dielectric screening in solvents with explicit expressions for the screening energy and its gradient. J. Chem. Soc. Perkin Trans. 1993, 2, 799-805. [CrossRef]

39. MOPAC Manual. Available online: http://OpenMOPAC.net/Manual (accessed on 10 January 2015).

Sample Availability: Samples of the compounds are not available from the authors.

(C) 2016 by the authors; licensee MDPI, Basel, Switzerland. This article is an open access article distributed under the terms and conditions of the Creative Commons by Attribution (CC-BY) license (http://creativecommons.org/licenses/by/4.0/). 